\title{
Form discrimination as a function of sex, procedure and tactual mode
}

GLEN M. VAUGHT

ALBION COLLEGE

Forty males and 40 females were randomly assigned to two tactual form discrimination tasks, easy and difficult under conditions of active and passive touch. Females were found to be better form discriminators than males and increased task difficulty resulted in highlighting female superiority.

When the hand is used to feel an object in the absence of vision, as in tactual form discrimination, it can be observed to make distinctive movements. Gibson(1966) described this-movement and called attention to its similarity with "visual scanning" (p. 215). Moreover, Gibson concluded that "evidently, exploratory movements of the fingers can be substituted for a grasping posture of the fingers, and the information about convexities and concavities can still be picked up" (p. 253). It would appear that two modes of tactual exploration emerge from Gibson's observations. The former suggests active manipulation of the tactual form while the latter suggests a more restricted mode of form manipulation; however, both modes make full use of the fingers as manipulators and both yield similar kinds of tactual information. Thus the fingers are important detectors of tactual information whether "grasping" or actively exploring a given object in the absence of "intended grasping."

It is, of course, well known that the fingers are important sensors and that the first two phalanges are particularly useful in discriminating among various objects in the environment. In recent pilot work, the author observed the importance of the first two phalanges of the exploring hand during form discrimination. In the course of this pilot work, additional Ss were run through the discrimination task without the use of the first two phalanges of their "'feeling" hand. Eliminating the first two phalanges via verbal instructions increased task difficulty and resulted in more discrimination errors than did previous procedures. Active-passive touch was not included in this preliminary work. Of course, one might expect to find Ss making more errors in this more difficult procedure relative to the easier condition in which the $S$ is able to employ the whole hand in exploration of the form.

Perhaps the most interesting observation from the above investigation was that females seemingly did better than males; i.e., females made fewer discrimination errors when deprived of the use of the first two phalanges of their discriminating hand. The purpose of the present study was to further explore sex differences in tactual form discrimination by restricting Ss from using the first two phalanges of their feeling hand in both active and passive touch conditions.

\section{Method}

The Ss were 40 male and 40 female introductory psychology students from Albion College, Albion, Michigan. Of these $80 \mathrm{Ss}, 20$ males and 20 females were randomly assigned to the two form discrimination conditions, easy and difficult. The difficult condition was defined by not allowing Ss to use the first two phalanges of their exploring hand during form discrimination trials, while in the easy condition, Ss had no such restrictions. The easy condition corresponded to the procedure normally used by the author in prior studies. In addition, all Ss were given both active and passive discrimination trials.

The forms and the procedure were essentially the same as those reported in previous studies (Vaught \& Augustson, 1967a, b; and Vaught \& Ellinger, 1966). The stability of this procedure has been established and reported elsewhere (Vaught, in press). Since all Ss received active and passive trials under both easy and difficult conditions, the procedure had to be slightly varied to allow for restrictions imposed on the Ss by depriving them of the full use of their hand during the difficult trials. The major stimulus presentation problem arose in the difficult condition (phalanges deprived) during active touch discrimination trials. This problem was solved by raising the form approximately 6 in. from the table and by E guiding the S's hand to the form. E also verbally instructed the $S$ regarding the form placement in his hand and was careful not to allow the $\mathrm{S}$ to grasp the form with the first two phalanges. During the passive trials in the difficult condition, the forms were placed in the Ss hand without presentation problems.

The stimuli were six Masonite forms patterned after those employed by Gaydos (1956). Each form was reproduced in identical shape and size withblackink on white posterboard, and was numbered for purposes of identification. The forms were presented in an upside down box that was draped on one side by a cloth. Each condition in which the $S$ performed was explained, but he was not given knowledge of the results nor any information regarding the number of trials to be run.

Presentation of the form for each trial was randomized by arbitrarily picking a form out of a box containing all six forms. The trials were further balanced for each $S$ by presenting 12 trials under one condition, 24 under another, and 12 more $(N=48)$ under the first condition in an ABBA sequence. The Ss were balanced by alternating the ABBA sequence with a $B A A B$ sequence of trials for every other $S$. One $S$ would begin and end with 12 active trials in the ABBA sequence while the next $S$ would begin and end with 12 passive trials in a 

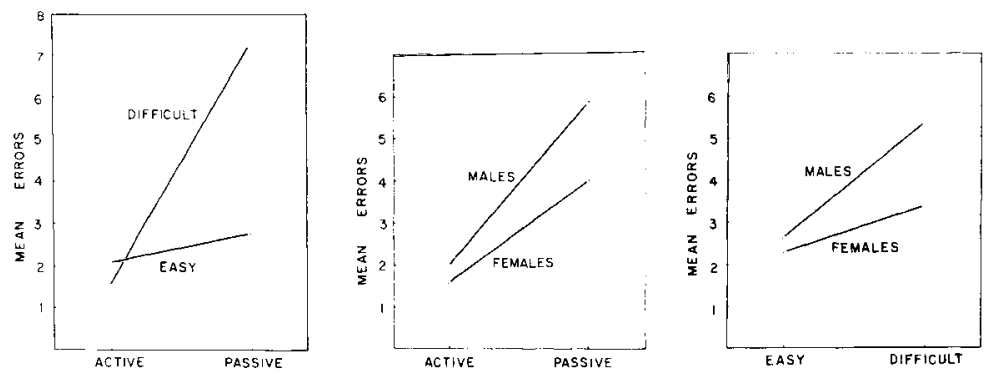

Fig. 1 (left). Procedure by tactual mode interaction for discrimination error means.

Fig. 2 (middle). Sex by tactual mode interaction for discrimination error means.

Fig. 3 (right). Sex by procedure interaction for discrimination error means.

BAAB sequence. These precautions were taken to control for learning by the Sand to control for the influence one particular sequence might have on another.

\section{Results and Discussion}

Analysis of variance was performed on the discrimination error scores for the three variables; i.e., sex, procedure (easy-difficult), and tactual mode (activepassive touch) with repeated measures on the latter variable. Depriving Ss of the first two phalanges of their exploring hand produced significantly more discrimination errors than did the easy condition where Ss were able to feel the form with their whole hand $(p<.01)$. Females made fewer errors than males $(p<$ $.01)$, and all Ss made more errors in passive touch than in active touch $(p<.01)$.

Figure 1 shows the significant interactions $(p<.01)$ for procedure and tactual mode. Many more discrimination errors were made during phalanges deprived trials than during easy trials in passive touch. This finding highlights the importance of the first two phalanges in tactual form discrimination, particularly in situations akin to passive touch where the $S$ is not actively engaged in touching. This finding also suggests that active participation in touch, in contrast to passive touch, in some way compensates for the loss of tactual information gained through the first two phalanges of the feeling hand.

The sex by tactual mode interaction was significant at the .05 level. As can be seen in Fig. 2, females made fewer errors than males in passive touch but not in active touch. Figure 3 shows the sex by procedure interaction which was also statistically significant $(p<.01)$. Once again females were observed to make fewer discrimination errors than males, this time when deprived of the use of the first two phalanges of their feeling hand.
The present findings have shown that individuals will habitually make more discrimination errors under conditions of passive touch. Furthermore, restricting Ss of the full use of their hands during discrimination trials results in more errors during passive touch performance. These results also show that sex differences in form discrimination are more likely to be observed in passive touch and that when task difficulty is increased, as in phalanges restriction, male-female differences are exacerbated. Thus, females appear to be better tactual form discriminators than males and for some reason a reduction in available stimuli succeeds in bringing out this difference.

These data might also be discussed in relation to what is known about sex differences in tasks involving manual dexterity. Tyler (1965) in a summary statement concerning the dexterity literature, stated that females are superior to males in tasks requiring "light, deft, swift movements of the hands (p. 246)." The present study also indicates that females are superior to males in tactual form discrimination tasks involving the availability of minimal amounts of sensory information from which to correctly identify a stimulus form.

\section{References}

GAYDOS, H. Intersensory transfer in the discrimination of form. Amer. J. Psychol., 1956, 69, 107-110.

GIBSON, J. J. The senses considered as perceptual systems. Boston: Houghton-Mifflin Co., 1966.

VAUGHT, G. M. Change in tactual form discrimination error scores as a function of vibratory stimulation. Psychon Sci., 1967, in press.

VAUGHT, G. M., \& AUGUSTSON, B. Field-dependence and form discrimination in females. Psychon. Sci, 1967a, 7, 333-334.

VAUGHT, G. M., \& AUGUSTSON, B. Field-dependence and form discrimination in males. Psychon. Sci. 1967b, 8, 233-234.

VAUGHT, G. M., \& ELLINGER, J. Field-dependence and form discrimination. Psychon. Sci, 1966, 6, 357-358.

TYLER, L. The psychology of human differences. (3rd ed.) New York: Appleton-Century-Crofts, 1965. 